\title{
Leucine-rich alpha-2 glycoprotein is a potential biomarker to monitor disease activity in inflammatory bowel disease receiving adalimumab: PLANET study
}

\author{
Shinichiro Shinzaki ${ }^{1}$ (D) Katsuyoshi Matsuoka ${ }^{2} \cdot$ Hiroki Tanaka $^{3} \cdot$ Fuminao Takeshima $^{4} \cdot$ \\ Shingo Kato ${ }^{5}$ - Takehiro Torisu ${ }^{6}$ - Yuki Ohta ${ }^{7}$ - Kenji Watanabe ${ }^{8}$. \\ Shiro Nakamura ${ }^{9}$ Naoki Yoshimura ${ }^{10}$ - Taku Kobayashi ${ }^{11}$ - Akiko Shiotani ${ }^{12}$. \\ Fumihito Hirai $^{13}$ - Sakiko Hiraoka ${ }^{14}$ - Mamoru Watanabe ${ }^{15}$ Minoru Matsuura ${ }^{16}$. $^{1}$ \\ Shohei Nishimoto $^{17} \cdot$ Shinta Mizuno $^{18} \cdot$ Hideki Iijima $^{1} \cdot$ Tetsuo Takehara $^{1} \cdot$ \\ Tetsuji Naka $^{19} \cdot$ Takanori Kanai $^{18} \cdot$ Takayuki Matsumoto $^{20}$
}

Received: 8 March 2021 / Accepted: 23 April 2021/Published online: 3 May 2021

(C) The Author(s) 2021

\begin{abstract}
Background This multicenter prospective study (UMIN000019958) aimed to evaluate the usefulness of serum leucin-rich alpha-2 glycoprotein (LRG) levels in monitoring disease activity in inflammatory bowel disease (IBD).
\end{abstract}

Shinichiro Shinzaki and Katsuyoshi Matsuoka contributed equally.

Supplementary Information The online version contains supplementary material available at https://doi.org/10.1007/s00535021-01793-0.

Shinichiro Shinzaki

shinzaki@gh.med.osaka-u.ac.jp

1 Department of Gastroenterology and Hepatology, Osaka University Graduate School of Medicine, 2-2 Yamadaoka, Suita, Osaka 565-0871, Japan

2 Inflammatory Bowel Disease Center, Toho University Sakura Medical Center, Chiba, Japan

3 IBD Center, Sapporo Kosei General Hospital, Hokkaido, Japan

4 Department of Gastroenterology and Hepatology, Nagasaki University Graduate School of Biomedical Sciences, Nagasaki, Japan

5 Department of Gastroenterology and Hepatology, Saitama Medical Center, Saitama Medical University, Saitama, Japan

6 Department of Medicine and Clinical Science, Graduate School of Medical Sciences, Kyushu University, Fukuoka, Japan

7 Department of Gastroenterology, Graduate School of Medicine, Chiba University, Chiba, Japan
Methods Patients with moderate-to-severe IBD initiated on adalimumab therapy were enrolled herein. Serum LRG, C-reactive protein (CRP), and fecal calprotectin (fCal) levels were measured at week $0,12,24$, and 52. Colonoscopy was performed at week 0,12 , and 52 for ulcerative colitis (UC), and at week 0, 24, and 52 for Crohn's disease (CD). Endoscopic activity was assessed using the Simple

8 Division of Internal Medicine, Center for Inflammatory Bowel Disease, Hyogo College of Medicine, Hyogo, Japan

9 Second Department of Internal Medicine, Osaka Medical College, Osaka, Japan

10 Department of Internal Medicine, Division of IBD, Tokyo Yamate Medical Center, Tokyo, Japan

11 Center for Advanced IBD Research and Treatment, Kitasato University Kitasato Institute Hospital, Tokyo, Japan

12 Division of Gastroenterology, Department of Internal Medicine, Kawasaki Medical School, Okayama, Japan

13 Department of Gastroenterology, Fukuoka University Faculty of Medicine, Fukuoka, Japan

14 Department of Gastroenterology and Hepatology, Okayama University Graduate School of Medicine, Dentistry and Pharmaceutical Sciences, Okayama, Japan

15 Department of Gastroenterology and Hepatology, Advanced Research Institute, Tokyo Medical and Dental University, Tokyo, Japan

16 Department of Gastroenterology and Hepatology, Kyorin University School of Medicine, Tokyo, Japan

17 Eisai Co. Ltd, Tokyo, Japan

18 Division of Gastroenterology and Hepatology, Department of Internal Medicine, Keio University School of Medicine, Tokyo, Japan 
Endoscopic Score for Crohn's Disease (SES-CD) for CD and the Mayo endoscopic subscore (MES) for UC.

Results A total of 81 patients was enrolled. Serum LRG levels decreased along with improvements in clinical and endoscopic outcomes upon adalimumab treatment $(27.4 \pm 12.6 \mu \mathrm{g} / \mathrm{ml}$ at week $0,15.5 \pm 7.7 \mu \mathrm{g} / \mathrm{ml}$ at week $12,15.7 \pm 9.6 \mu \mathrm{g} / \mathrm{ml}$ at week 24 , and $14.5 \pm 6.8 \mu \mathrm{g} / \mathrm{ml}$ at week 52), being correlated with endoscopic activity at each time point (SES-CD: $r=0.391$ at week $0, r=0.563$ at week 24, $r=0.697$ at week 52; MES: $r=0.534$ at week 0 , $r=0.429$ at week $12, r=0.335$ at week 52). Endoscopic activity better correlated with LRG compared to CRP and fCal on pooled analysis at all time points (SES-CD: LRG: $r=0.636$, CRP: $r=0.402$, fCal: $r=0.435$; MES: LRG: $r=0.568$, CRP: 0.389, fCal: $r=0.426$ ).

Conclusions Serum LRG is a useful biomarker of endoscopic activity both in CD and UC during the adalimumab treatment.

Keywords Crohn's disease - Ulcerative colitis - Leucinerich alpha-2 glycoprotein $\cdot$ Biomarker

\section{Introduction}

The etiology of inflammatory bowel disease (IBD), such as Crohn's disease (CD) and ulcerative colitis (UC), is still unknown, and the number of patients with these diseases is increasing worldwide $[1,2]$. Recent therapeutic progress has enabled us to select several therapeutic options for IBD, including anti-tumor necrosis factor (TNF) agents and immunosuppressants [3]. Using these medications in appropriate situations, the goal of the treatment has changed from clinical remission to mucosal healing, which has decreased the risks for hospitalization and surgical intervention.

The treat-to-target strategy requires frequent monitoring of disease activity. Recently updated STRIDE-II statements have mentioned that the most important long-term achievable treatment targets for IBD patients are clinical remission, endoscopic healing, restoration of quality of life, and absence of disability [4]. Colonoscopy is also the current gold standard for monitoring mucosal healing in IBD patients [5]. However, frequent endoscopy is difficult to perform, because it is time-consuming, invasive, and labor-intensive. Serum C-reactive protein (CRP) is widely used as a serum biomarker for predicting the clinical activity of inflammatory disorders, including IBD;

19 Center for Intractable Immune Disease, Kochi Medical School, Kochi University, Kochi, Japan

20 Division of Gastroenterology, Department of Medicine, Iwate Medical University, Iwate, Japan however, the CRP levels are not always elevated in active IBD patients [6]. Currently, fecal calprotectin (fCal) has become widely used as an accurate biomarker for mucosal healing of UC [7]. However, the correlation of fCal with clinical symptoms is fair to poor in UC [8], and the usefulness of $\mathrm{fCal}$ in $\mathrm{CD}$ remains unclear due to the variable accuracy. Moreover, the fecal samples have problems, such as difficulty in performing on-demand sampling, instability of samples, and prolonged time until obtaining test results in many institutions. Furthermore, patients often forget to present stool samples during their hospital visits. Therefore, to develop treat-to-target strategies for IBD, serum biomarkers more accurately reflecting endoscopic disease activity are necessary.

Through comprehensive proteomics analysis of serum samples from rheumatoid arthritis patients treated with infliximab, we identified serum leucine-rich alpha-2 glycoprotein (LRG) as a novel biomarker of disease activity of rheumatoid arthritis [9]. LRG is a 50-kDa glycoprotein first reported by Haupt et al. in 1977 [10], containing eight leucine-rich repeat domains; however, its physiological function remains unclear. We previously reported that the serum LRG levels were correlated with the disease activity in both $\mathrm{CD}$ and $\mathrm{UC}$ patients $[9,11]$. We have also demonstrated that the serum LRG levels were elevated in patients with active $\mathrm{CD}$ and $\mathrm{UC}$ with normal serum CRP levels $[9,11]$, and more strongly associated with mucosal healing of UC than the CRP level [12]. However, the correlation between serum LRG levels and endoscopic activity remains unknown. In addition, the usefulness of LRG in monitoring the disease activity of IBD during induction and maintenance therapy is unclear. Moreover, these studies are retrospective, and no prospective studies have been performed to investigate the clinical usefulness of LRG in predicting the clinical activity of IBD.

This prospective observational study (Predictor and biomarker: leucine-rich alpha-2 glycoprotein for inflammatory bowel disease treatment with adalimumab [PLANET]) aimed to investigate the association of serum LRG levels with disease activity and endoscopic activity in IBD patients treated with adalimumab for 52 weeks, thus examining the usefulness of LRG as a monitoring biomarker during induction and maintenance therapy.

\section{Methods}

\section{Patients}

This multicenter prospective observational study was conducted between January 2016 and March 2018 at 20 institutions throughout Japan. This study was registered 
with the University hospital Medical Information Network (UMIN000019958).

Patients initiated on adalimumab therapy were recruited and evaluated for 52 weeks or until treatment discontinuation. The inclusion criteria were as follows: age $\geq 15$ years, having moderate-to-severe active IBD defined by a CD activity index (CDAI) score of $\geq 220$ in CD or a Mayo score of $\geq 6$ in UC [13], and displaying inadequate responses to conventional therapies including corticosteroids and immunomodulators. CD and UC were diagnosed on the basis of established diagnostic criteria [13]. Exclusion criteria included bowel resection within 12 weeks, patients with stoma or ileal pouch (excluding ileo-rectal anastomosis), prior exposure to adalimumab, history of primary non-response to infliximab or secondary non-response to infliximab at $10 \mathrm{mg} / \mathrm{kg}$, and contraindications to adalimumab.

\section{Treatment}

Adalimumab was administered at $160 \mathrm{mg}$ subcutaneously at week 0 , followed by $80 \mathrm{mg}$ at week 2 , and then $40 \mathrm{mg}$ at every other week. In CD, the dose could be increased to $80 \mathrm{mg}$ if response was lost.

The following drugs were permitted to be used concomitantly if the dosage was stabilized during the 4 weeks before the study and maintained until the end of the study. The dose of the following medications could be reduced in accordance with the patients' condition; 5-aminosalysalicylic acid, azathioprine, 6-mercaptopurine, methotrexate, oral corticosteroids, antibiotics for $\mathrm{CD}$, and enteral nutrition.

Concomitant use of biologics other than adalimumab, intravenous corticosteroids, rectal corticosteroids or 5-aminosalicylic acid, ciclosporin, tacrolimus, cytaphresis, and total parenteral nutrition was prohibited throughout the study.

\section{Study design}

Patient visits were scheduled upon administration of the first dose of adalimumab (week 0), week 12, 24, and 52 after initiating adalimumab treatment. Serum LRG levels, fCal levels, CDAI scores in CD or Partial Mayo scores (PMS) in UC were evaluated at week 0, 12, 24, and 52. If a patient discontinued the study before week 52 , these variables were assessed upon discontinuation. Serum LRG levels were measured with a NANOPIA ${ }^{\circledR}$ LRG Kit based on the latex turbidimetry method (Sekisui Medical, Tokyo, Japan). Colonoscopy was performed at baseline (within 4 weeks prior to adalimumab initiation) and at week 12 , and 52 for UC, and at baseline, week 24 and 52 for CD. Endoscopic activity was assessed as the Simple Endoscopic
Score for Crohn's disease (SES-CD) in CD [14] or the Mayo endoscopic subscore (MES) in UC [15]. Endoscopic activity was scored by on-site investigators. Clinical remission was defined by a CDAI score of $<150$ or a PMS of $\leq 2$; Endoscopic remission was defined by a SES-CD of $<4$ [16] or a MES of $\leq 1$ [17].

The following variables were recorded: age, sex, height, weight, smoking, concomitant medication, history of biologics (drug name, period of use, and reason for discontinuation), disease duration, disease location based on Montreal classification criteria [18], history of bowel surgery (time and type of surgery), and the presence or absence of perianal lesions (fissuring ulcer, fistula, abscess, etc.).

\section{Endpoints}

The primary endpoints of this exploratory study are the following: (1) changes in adalimumab treatment-related biomarkers and (2) the correlation between the biomarker levels and endoscopic activities.

\section{Statistical analysis}

Continuous variables are presented as mean \pm standard deviation (SD) values, and non-parametric ones as median with range. The Wilcoxon signed rank test was performed to compare non-parametric paired values, and the paired $t$ test was performed to compare parametric paired values. Between-group differences were analyzed using the Kruskal-Wallis test for non-parametric data and Student's $t$ test for parametric values. Categorical values are presented as numbers $(\%)$ and differences were analyzed using the Chi square test or Fisher's exact test. Pearson's test was performed to analyze the association between biomarkers and activity indices. Values of $p<0.05$ were considered statistically significant.

\section{Results}

\section{Patients}

A total of 81 patients (34 CD, $47 \mathrm{UC}$ ) were enrolled herein, and their background characteristics are summarized in Table 1. Eighty patients were enrolled as the full analysis set, and 56 patients $(69.1 \%)$ completed 52 weeks of treatment (Supplementary Fig. 1). 
Table 1 Baseline patient characteristics $(n=81)$

\begin{tabular}{ll}
\hline Sex, female/male, $n(\%)$ & $30(37.0) / 51(63.0)$ \\
Age, years, median (range) & $35.9(15-70)$ \\
Body mass index, mean (SD) & $20.1(3.3)$ \\
Disease, CD/UC, $n(\%)$ & $34(42.0) / 47(58.0)$ \\
Disease duration, years, mean (SD) & $5.9(6.7)$ \\
Disease location in CD, ileitis/colitis/ileocolitis, $n(\%)$ & $3(8.8) / 4(11.8) / 27(79.4)$ \\
Disease location in UC, left-sided/extensive, $n(\%)$ & $16(34.0) / 31(66.0)$ \\
Perianal lesion in CD, $n(\%)$ & $21(61.8)$ \\
Concomitant medications & \\
5-aminosalycilic acid, $n(\%)$ & $70(86.4)$ \\
Thiopurine, $n(\%)$ & $29(35.8)$ \\
Corticosteroid, $n(\%)$ & $43(53.1)$ \\
Previous use of biologics, $n(\%)$ & $8(9.9)$ \\
History of bowel surgery in CD, $n(\%)$ & $8(23.5)$ \\
Current smoker, $n(\%)$ & $3(3.7)$ \\
Laboratory data & \\
LRG ( $\mu \mathrm{g} / \mathrm{ml}), n=80$, mean $(\mathrm{SD})$ & $27.4(12.6)$ \\
CRP (mg/dl), $n=80$, mean (SD) & $1.69(2.29)$ \\
fCal $(\mu \mathrm{g} / \mathrm{g}), n=71$, mean $(\mathrm{SD})$ & $5867(6766)$ \\
CDAI in CD, mean (SD) & $271.5(55.5)$ \\
SES-CD in CD, $n=33$, mean $(\mathrm{SD})$ & $17.3(7.5)$ \\
PMS in UC, mean $(\mathrm{SD})$ & $5.9(1.3)$ \\
MES in UC, $n=45$, mean $(\mathrm{SD})$ & $2.3(0.6)$ \\
\hline$S D$ stand $(\%)$ &
\end{tabular}

$S D$ standard deviation, $L R G$ leucine-rich alpha-2 glycoprotein, CRP C-reactive protein, CDAI Crohn's disease activity index, $S E S$-CD Simplified Endoscopic Score for Crohn's disease, $P M S$ partial Mayo score, MES Mayo endoscopic subscore
Fig. 1 Changes in leucine-rich alpha-2 glycoprotein (LRG), C-reactive protein (CRP), and fecal calprotectin (fCal) during adalimumab treatment of IBD patients. Data were obtained before treatment (week 0 ) and at 12,24 , and 52 weeks after the treatment and shown in mean \pm standard deviation (SD). ${ }^{* *} p<0.001, * p<0.01$ vs. week 0 by paired $t$ test

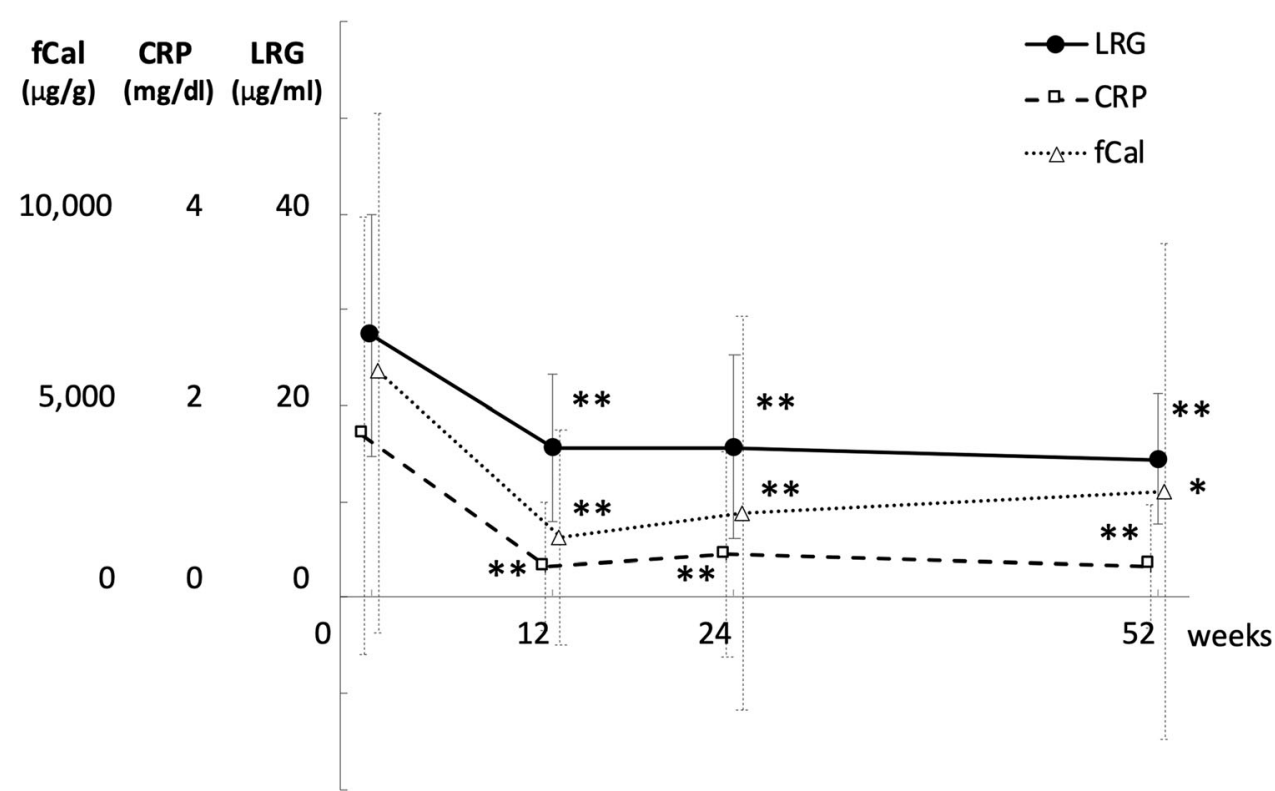

\section{Changes in disease activity upon initiation of adalimumab therapy}

Given that this study was focused on the alteration of biomarkers by adalimumab treatment, we first analyzed the clinical and endoscopic efficacies among patients who continued adalimumab in each evaluation point. In total, $65.2 \%(45 / 69)$ of patients achieved clinical remission at week 12; week 24, 69.2\% (45/65); week 52, 69.1\% (38/55). Endoscopic remission was achieved in $44.2 \%(23 / 52)$ of 
patients at week 52 (Supplementary Fig. 2). In CD, both clinical and endoscopic activities significantly decreased upon initiation of adalimumab treatment (CDAI; 271.5 at week $0,128.0$ at week $12 ; p<0.0001,133.4$ at week 24 ; $p<0.0001$, and 115.9 at week $52 ; p<0.0001$ vs. week 0 , SES-CD; 17.3 at week $0,10.1$ at week $24 ; p<0.0001$, and 8.6 at week 52; $p<0.0001$ vs. week 0) (Supplementary Fig. 3a). Also, the clinical and endoscopic activities significantly decreased upon initiation of adalimumab treatment for UC (PMS; 5.9 at week 0, 2.0 at week 12; $p<0.0001,1.4$ at week $24 ; p<0.0001$, and 1.7 at week 52; $p<0.0001$ vs. week 0 , MES; 2.30 at week $0,1.20$ at week $12 ; p<0.0001$, and 1.2 at week $52 ; p<0.0001$ vs. week 0) (Supplementary Fig. 3b).

\section{Decreased serum LRG, CRP, and fCal levels with adalimumab treatment}

Concurrent with clinical and endoscopic improvements, serum LRG levels significantly decreased from $27.4 \pm 12.6 \mu \mathrm{g} / \mathrm{ml}$ at week $0(n=80)$ to $15.5 \pm 7.7 \mu \mathrm{g} / \mathrm{ml}$ at week $12(n=70, p<0.0001$ vs. week 0$)$, $15.7 \pm 9.6 \mu \mathrm{g} / \mathrm{ml}$ at week $24(n=64, p<0.0001$ vs. week $0)$, and $14.5 \pm 6.8 \mu \mathrm{g} / \mathrm{ml}$ at week $52(n=55, p<0.0001$ vs. week 0). Serum CRP levels also significantly decreased from $1.69 \pm 2.29 \mathrm{mg} / \mathrm{dl}$ at week 0 to $0.33 \pm 0.67 \mathrm{mg} / \mathrm{dl}$ at week $12(p<0.0001$ vs. week 0$), 0.45 \pm 1.08 \mathrm{mg} / \mathrm{dl}$ at week 24 ( $p<0.0001$ vs. week 0$)$, and $0.33 \pm 0.65 \mathrm{mg} / \mathrm{dl}$ at week $52(p<0.0001$ vs. week 0$)$, and fCal levels significantly decreased from $5867 \pm 6766 \mu \mathrm{g} / \mathrm{g}$ at week 0 to $1574 \pm 2790 \mu \mathrm{g} / \mathrm{g} \quad$ at week $12 \quad(p<0.0001)$, $2193 \pm 5120 \mu \mathrm{g} / \mathrm{g}$ at week $24(p=0.0017$ vs. week 0$)$, and $2763 \pm 6463 \mu \mathrm{g} / \mathrm{g}$ at week $52(p=0.036$ vs. week 0$)$ (Fig. 1). We also confirmed that the LRG, CRP and fCal levels were significantly decreased at 12, 24, and 52 weeks when the patients were divided into CD and UC (Supplementary Fig. 4).

\section{LRG, CRP, and fCal levels at each time point in patients with and without endoscopic remission at week 52}

LRG, CRP, and fCal levels at each time point were stratified by endoscopic remission at week 52 (Fig. 2). The mean LRG levels at week $0.12,24$, and 52 were significantly lower in patients with endoscopic remission at week 52 compared to those who did not (week $0 ; 23.4 \mu \mathrm{g} / \mathrm{ml}$ vs. $31.6 \mu \mathrm{g} / \mathrm{ml} ; p=0.0244$, week $12 ; 11.2 \mu \mathrm{g} / \mathrm{ml}$ vs. $18.1 \mu \mathrm{g} /$ $\mathrm{ml} ; p<0.0001$, week $24 ; 10.8 \mu \mathrm{g} / \mathrm{ml}$ vs. $20.0 \mu \mathrm{g} / \mathrm{ml}$; $p=0.0002$, week $52 ; 11.7 \mu \mathrm{g} / \mathrm{ml} \quad$ vs. $17.1 \mu \mathrm{g} / \mathrm{ml}$; $p=0.026$ ). The mean CRP level was significantly lower in patients with endoscopic remission than those who did not at week $0,12,24$, but not at week 52 (week $0 ; 1.04 \mathrm{mg} / \mathrm{dl}$
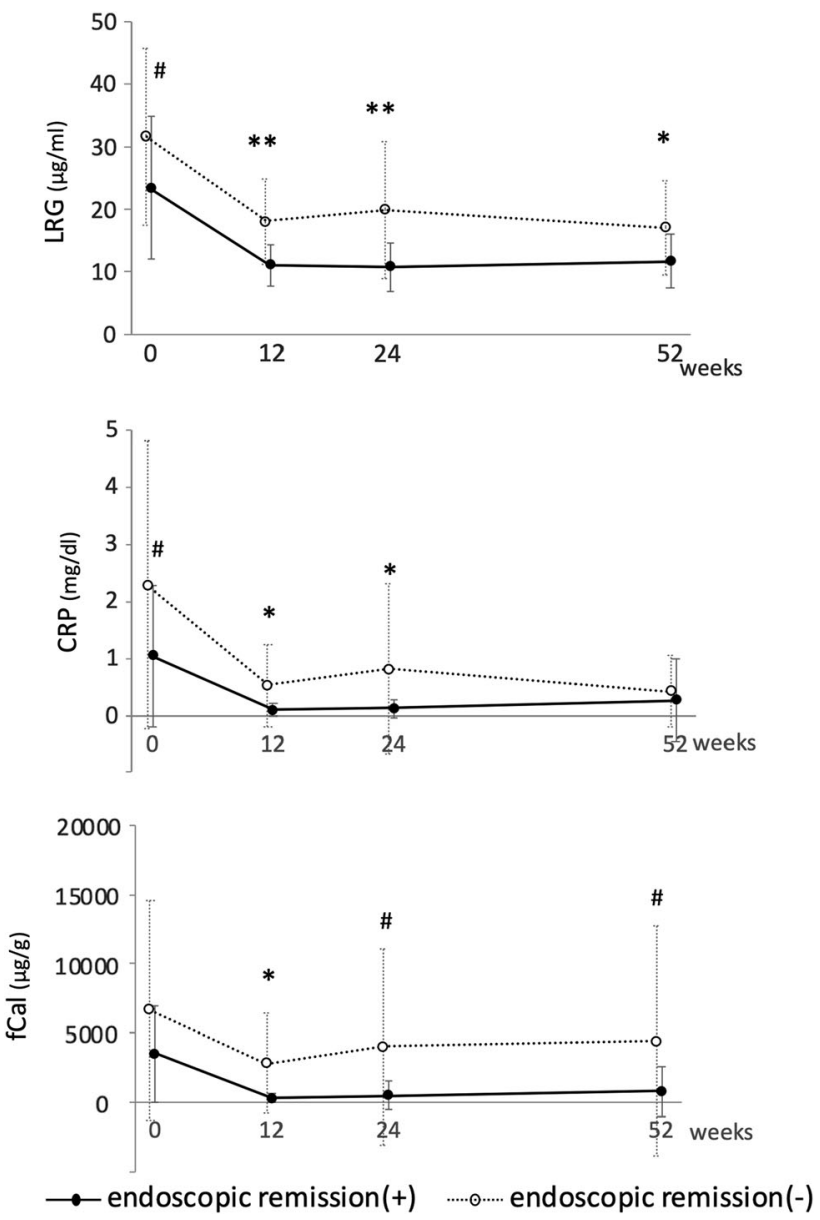

Fig. 2 Changes in the biomarkers in IBD patients before and after the adalimumab treatment depending on the response to treatment. Levels of LRG, CRP, and fCal in patients with endoscopic remission at week $52(n=23$, solid line) and those without $(n=29$, dotted line) were shown in mean $\pm \mathrm{SD}$ and compared in each time point. $* * p<0.001$, $* p<0.01$, \# $p<0.05$ by Student's $t$ test

vs. $2.28 \mathrm{mg} / \mathrm{dl} ; p=0.0251$, week $12 ; 0.10 \mathrm{mg} / \mathrm{dl}$ vs. $0.53 \mathrm{mg} / \mathrm{dl} ; p=0.0033$, week $24 ; 0.12 \mathrm{mg} / \mathrm{dl}$ vs. $0.82 \mathrm{mg} /$ $\mathrm{dl} ; p=0.0175$, week $52 ; 0.26 \mathrm{mg} / \mathrm{dl}$ vs. $0.42 \mathrm{mg} / \mathrm{dl}$; $p=0.3941)$. Also, the mean fCal level was significantly lower in patients with endoscopic remission than those who did not at week 12, 24, 52, but not at week 0 (week 0; $3492 \mu \mathrm{g} / \mathrm{g}$ vs. $6632 \mu \mathrm{g} / \mathrm{g} ; p=0.0767$, week $12 ; 267 \mu \mathrm{g} / \mathrm{g}$ vs. $2798 \mu \mathrm{g} / \mathrm{g} ; p=0.0011$, week $24 ; 481 \mu \mathrm{g} / \mathrm{g}$ vs. $4024 \mu \mathrm{g} /$ $\mathrm{g} ; \quad p=0.0124$, week $52 ; 796 \mu \mathrm{g} / \mathrm{g} \quad$ vs. $4433 \mu \mathrm{g} / \mathrm{g}$; $p=0.3941)$.

\section{Correlation between disease activity and biomarkers}

We assessed the correlation between disease activity and biomarker levels at each time point (Table 2). In CD, LRG levels were correlated with CDAI scores at weeks 12, 24 and 52. Furthermore, CRP levels were correlated with CDAI scores at weeks 0,24 , and 52, while fCal levels were 
Table 2 Correlation of serum and fecal biomarkers with clinical disease activity

\begin{tabular}{|c|c|c|c|c|c|c|c|c|c|c|c|c|c|}
\hline & & \multicolumn{3}{|c|}{ Week 0} & \multicolumn{3}{|c|}{ Week 12} & \multicolumn{3}{|c|}{ Week 24} & \multicolumn{3}{|c|}{ Week 52} \\
\hline & & $n$ & $r$ & $p$ & $n$ & $r$ & $p$ & $n$ & $r$ & $p$ & $n$ & $r$ & $p$ \\
\hline \multirow[t]{3}{*}{ CDAI (CD) } & LRG & 34 & 0.233 & 0.1851 & 33 & 0.440 & 0.0105 & 33 & 0.743 & $<0.0001$ & 28 & 0.554 & 0.0022 \\
\hline & CRP & 34 & 0.375 & 0.0287 & 33 & 0.339 & 0.0540 & 33 & 0.622 & 0.0001 & 28 & 0.597 & 0.0008 \\
\hline & fCal & 32 & -0.097 & 0.5982 & 31 & 0.014 & 0.9387 & 32 & 0.204 & 0.2616 & 27 & 0.600 & 0.0009 \\
\hline \multirow[t]{3}{*}{ PMS (UC) } & LRG & 46 & 0.503 & 0.0004 & 36 & 0.402 & 0.0151 & 31 & 0.376 & 0.0368 & 27 & 0.398 & 0.0398 \\
\hline & CRP & 46 & 0.440 & 0.0022 & 35 & 0.372 & 0.0280 & 32 & 0.441 & 0.0115 & 27 & 0.072 & 0.7204 \\
\hline & fCal & 39 & 0.108 & 0.5117 & 36 & 0.411 & 0.0127 & 31 & 0.327 & 0.0730 & 24 & 0.494 & 0.0141 \\
\hline
\end{tabular}

CDAI Crohn's disease activity index, PMS partial Mayo score, $L R G$ leucine-rich alpha-2 glycoprotein, $C R P$ C-reactive protein, $f C a l$ fecal calprotectin

significantly correlated with CDAI scores only at week 52 . In UC, LRG levels were also correlated with PMS scores at each time point. CRP levels were correlated with PMS scores at weeks 0,12 , and 24, while fCal levels were significantly correlated with PMS scores only at week 12 and 52.

\section{Correlation between endoscopic activity and biomarkers}

We next evaluated the correlation between endoscopic activity and biomarker levels at each time point (Table 3 ). In CD, LRG levels were correlated with SES-CD at each time point, while CRP levels were correlated with SES-CD only at week 24 and fCal levels at weeks 24 and 52. In UC, LRG levels were also correlated with MES at weeks 0 and 12 , while CRP levels were correlated with MES only at week 0 and fCal levels at week 12 .

Each biomarker was assessed at all time points together to evaluate the correlation of each biomarker with clinical activity (Supplementary Fig. 5) and endoscopic activity (Fig. 3). Among the three biomarkers, LRG was most significantly correlated with both clinical activity indices (CDAI and PMS) and endoscopic activity indices (SES-CD and MES).

\section{Discussion}

Herein, we assessed LRG, CRP, fCal and disease activity indices serially during a 52-week period among IBD patients treated with adalimumab for induction and maintenance of remission. LRG levels reflected clinical and endoscopic disease activity at each predetermined time point. The correlation between LRG and disease activity was superior to that of CRP and $\mathrm{fCal}$ at each time point. LRG levels better correlated with endoscopic activity than CRP and fCal, indicating that LRG is a potential surrogate marker for endoscopic activity and useful for monitoring disease activity during the induction and maintenance of remission in IBD.

Ideal biomarkers are simple, easy to screen, noninvasive, cost-effective, rapid, and reproducible [6]. Considering these biomarker requirements, LRG has several

Table 3 Correlation of serum and fecal biomarkers with endoscopic disease activity

\begin{tabular}{|c|c|c|c|c|c|c|c|c|c|c|c|c|c|}
\hline & & \multicolumn{3}{|c|}{ Week 0} & \multicolumn{3}{|c|}{ Week 12} & \multicolumn{3}{|c|}{ Week 24} & \multicolumn{3}{|c|}{ Week 52} \\
\hline & & $n$ & $r$ & $p$ & $n$ & $r$ & $p$ & $n$ & $r$ & $p$ & $n$ & $r$ & $p$ \\
\hline \multirow[t]{3}{*}{ SES-CD (CD) } & LRG & 33 & 0.391 & 0.0244 & - & - & - & 27 & 0.563 & 0.0022 & 25 & 0.697 & 0.0001 \\
\hline & CRP & 33 & 0.107 & 0.5536 & - & - & - & 27 & 0.470 & 0.0134 & 25 & 0.384 & 0.0582 \\
\hline & fCal & 31 & 0.300 & 0.1005 & - & - & - & 26 & 0.659 & 0.0003 & 25 & 0.593 & 0.0398 \\
\hline \multirow[t]{3}{*}{ MES (UC) } & LRG & 45 & 0.534 & 0.0002 & 31 & 0.429 & 0.0159 & - & - & - & 27 & 0.335 & 0.0877 \\
\hline & CRP & 45 & 0.538 & 0.0001 & 30 & 0.234 & 0.2137 & - & - & - & 27 & -0.022 & 0.9134 \\
\hline & fCal & 38 & 0.194 & 0.2430 & 31 & 0.448 & 0.0116 & - & - & - & 24 & 0.393 & 0.0578 \\
\hline
\end{tabular}

SES-CD Simplified Endoscopic Score for Crohn's disease, MES Mayo endoscopic subscore, LRG leucine-rich alpha-2-glycoprotein, CRP C-reactive protein, $f C a l$ fecal calprotectin 

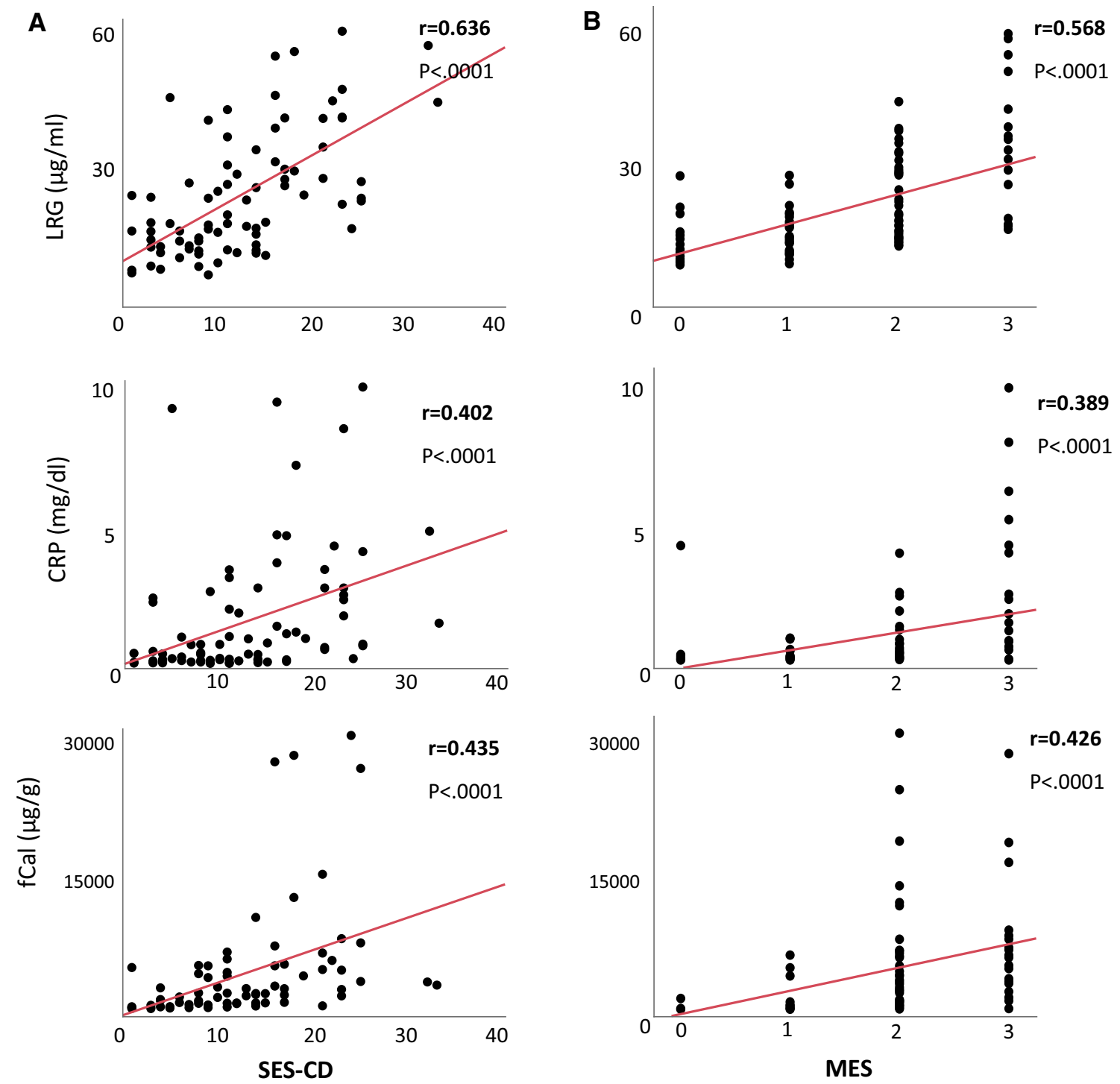

Fig. 3 Correlation of serum and fecal biomarkers with endoscopic activities. All serial time points were put into an analysis, and the correlation of biomarkers with (a) simple endoscopic score for Crohn's disease (SES-CD) in CD or (b) Mayo endoscopic subscore (MES) in UC are shown

advantages over other preclinical serum biomarkers reportedly used to assess IBD disease activity [19, 20]. A commercial LRG assay has been approved and is available for medical use in Japan, yielding results in $10 \mathrm{~min}$. Thus, LRG can be assessed in daily clinical practice.

The treat-to-target strategy has been introduced for IBD treatment. The CALM trial assessed the effects of this strategy in CD [21]. This study shows that additional assessment of CRP and fCal along with symptoms to facilitate decisions regarding treatment modifications improves endoscopic remission rates at 52 weeks after adalimumab initiation. These results show the importance of monitoring disease activity during induction therapy through objective analysis. Thus far, CRP and fCal have been assessed as objective disease activity biomarkers for
IBD during the clinical management of IBD [4]. However, serum CRP levels may not be elevated even in patients with active mucosal inflammation [22]. Although fCal is correlated with mucosal inflammatory activity in UC [23], limited information is available regarding whether it is correlated with disease activity during small intestinal mucosal inflammation in CD [24-26]. Furthermore, stool sampling is troublesome for patients. Therefore, serum biomarkers more accurately reflecting the endoscopic activity of IBD are warranted.

Herein, LRG displayed a higher correlation with endoscopic activity than CRP, probably because CRP is produced by hepatocytes solely in response to interleukin (IL)6 stimulation, whereas LRG is induced not only by IL- 6 but also by several other proinflammatory cytokines including 
IL-22 and TNF [11]. Furthermore, LRG is produced by intestinal epithelial cells [11], thus potentially reflecting inflammatory activity more sensitively.

We also clarified that LRG was significantly lower in patients with endoscopic remission at week 52 than those without at all time points, but CRP and fCal showed no differences at week 52 and week 0 , respectively (Fig. 2). This indicates that LRG can stably predict the long-term endoscopic response at week 52 during the adalimumab treatment course. Given that TNF is one of the cytokines that drive LRG production [11], theoretically, anti-TNF treatment can influence the serum LRG levels. However, our results clearly showed that LRG was strongly associated with the disease activity indices, even during the treatment with adalimumab. These results suggest that LRG can be useful for monitoring the disease activity, even after the anti-TNF therapy.

The present results support the use of LRG during induction therapy for IBD as follows: LRG levels can be determined before treatment initiation for induction of remission and then measured periodically to monitor changes in disease activity. If LRG levels do not decrease, treatment should be intensified. If LRG levels are within normal levels, colonoscopy should be performed to confirm mucosal healing, which is currently the most reliable therapeutic goal, and maintenance therapy can be continued. The cutoff value of LRG, like fCal, can vary depending on the disease location and clinical manifestation, and further investigation is required.

This study has several limitations. First, we are unsure whether the present results may be generalized to IBD therapies other than adalimumab, because we only enrolled patients receiving adalimumab therapy. Second, histological analysis has not been performed in the present study, because our aim was to consolidate the usefulness of LRG in monitoring mucosal healing prospectively. Third, it was unclear whether the presence of extra-intestinal manifestations, perianal fistula and deep small bowel lesions affected LRG levels. A larger study, including patients receiving various induction therapies and subjected to another activity indices including histological analysis, is needed to clarify these issues.

In conclusion, this study shows that LRG, a novel serum biomarker, better reflects endoscopic activity during adalimumab treatment than CRP or fCal among IBD patients, indicating that LRG can be considered a potential biomarker to monitor disease activity during induction and maintenance therapy and can be incorporated in the treatto-target strategy for the clinical management of IBD.

Acknowledgements Guarantor of the article: Shinichiro Shinzaki, $\mathrm{MD}, \mathrm{PhD}$ accepts full responsibility for the conduct of the study.
Author contributions SS, KM, SNi, SM, HI, TN, TKa, TM planned and conducted the study. SS, HT, FT, SK, TTo, YO, SNa, KW, NY, TKo, AS, FH, SH, MW, MM, SM, KM collected the data, SS, HI, SM, KM, TTa, TN, TKa, TM interpreted the data, SS, KM, HI, TN, TM drafted the manuscript. Each author has approved the final draft submitted.

Funding This study was supported by Eisai Co., Ltd. (Tokyo, Japan). Eisai Co., Ltd. was not involved in the study design, data collection, or interpretation of data. Statistical analysis was performed by RPM Co. Ltd. (Tokyo, Japan).

Data availability The data underlying this article will be shared on reasonable request to the corresponding author.

\section{Declarations}

Conflict of interest KM reports personal fees from EA Pharma Co., Ltd., Mitsubishi Tanabe Pharma, Takeda Pharmaceutical Co. Ltd., Janssen Pharmaceutical K.K., Abbvie Inc., Mochida Pharmaceutical Co., Ltd., Kissei Pharmaceutical Co., Ltd., Alfressa Pharma Corporation, JIMRO Co., Ltd.; research grant from Janssen Pharmaceutical K.K., outside the submitted work. HT reports personal fees from JIMRO Co., Ltd., AbbVie GK, EA Pharma Co., Ltd., Mochida Pharmaceutical Co., Ltd., Kyorin Pharmaceutical Co., Ltd., outside the submitted work. SK reports personal fees from Abbvie Inc., Mitsubishi Tanabe Pharma Corporation, Janssen Pharmaceutical K.K.; grants from Abbvie Inc., EA Pharma Co., Ltd., outside the submitted work. TTo reports grants from Mitsubishi Tanabe Pharma Corporation, Abbvie Inc., EA Pharma Co., Ltd., outside the submitted work. KW reports grants and personal fees from AbbVie Japan Co., Ltd., EA Pharma Co., Ltd., Mitsubishi Tanabe Pharma Corporation, Takeda Pharmaceutical Co., Ltd., Kyorin Pharmaceutical Co., Ltd., Pfizer Japan Inc., Kissei Pharmaceutical Co., Ltd., outside the submitted work; endowed chair from AbbVie Japan Co., Ltd., EA Pharma Co., Ltd., Mitsubishi Tanabe Pharma Corporation, Kyorin Pharmaceutical Co., Ltd., Zeria Pharmaceutical Co., Ltd., JIMRO Co Ltd., Mochida Pharmaceutical Co., Ltd., Asahi Kasei Medical Co., Ltd., Otsuka Pharma Factory Inc. SNa reports personal fees and endowed chair from Mitsubishi Tanabe Pharma Corporation, Abbvie Inc., EA Pharma Co., Ltd., Janssen Pharmaceutical K.K., Mochida Pharmaceutical Co., Ltd., Takeda Pharmaceutical Co. Ltd., Kyorin Holdings Inc., JIMRO Co Ltd., Zeria Pharmaceutical Co., Ltd.; research grants from Mitsubishi Tanabe Pharma Corporation, Mochida Pharmaceutical Co., Ltd., Takeda Pharmaceutical Co. Ltd., endowed chair from Otsuka Pharma Factory Inc., outside the submitted work. YN reports personal fees from Janssen Pharmaceutical K.K., Mitsubishi Tanabe Pharma Corporation, Mochida Pharmaceutical Co., Ltd., outside the submitted work. TKo reports personal fees from Alfresa Pharma, Mochida Pharmaceutical, Takeda Pharmaceutical, Mitsubishi Tanabe Pharma, Janssen, Abbvie GK, Pfizer; grants from Abbvie GK, EA Pharma, Otsuka Holdings, ZERIA, EA Pharma, Nippon Kayaku, JIMRO, outside the submitted work. AK reports personal fees from Takeda Pharmaceutical Co., Ltd., Astellas Pharma Inc., Daiichisankyo Co., Ltd.; research grants from Takeda Pharmaceutical Co., Ltd., Mochida Pharmaceutical Co., Ltd., AstraZeneca K. K., Daiichisankyo Co., Ltd., outside the submitted work. FH reports personal fees from Abbvie GK, EA Pharma Co., Ltd, Janssen Pharmaceutical K.K, Mochida Pharmaceutical Co., Ltd., Takdeda Pharmaceutical Co., Ltd and Mitsubishi Tanabe Pharma, outside the submitted work. SH reports personal fees from Janssen Pharmaceutical K.K., Mitsubishi Tanabe Pharma Corporation, outside the submitted work. WM reports personal fees from Mitsubishi Tanabe Pharma Corporation, Takeda Pharmaceutical Co., Ltd., Zeria Pharmaceutical Co., Ltd., Pfizer Japan Inc., Janssen Pharmaceutical K.K., 
Gilead Sciences, Inc., EA Pharma Co., Ltd.; research grants from Alfresa Pharma Corporation.; scholarship grants from Abbvie GK., Mitsubishi Tanabe Pharma Corporation, Takeda Pharmaceutical Co., Ltd., Zeria Pharmaceutical Co., Ltd., Astellas Pharma Inc., Taiho Pharmaceutical Co., Ltd., Nippon Kayaku Co., Ltd., Mochida Pharmaceutical Co., Ltd., Miyarisan Pharmaceutical Co., Ltd., Kyorin Pharmaceutical Co., Ltd., EA Pharma Co., Ltd., Kissei Pharmaceutical Co., Ltd., Alfresa Pharma Corporation., outside the submitted work. MM reports personal fees from Janssen Pharmaceutical K.K. and grants from Nippon Kayaku Co., Ltd., outside the submitted work. SNi is an employee of Eisai Co. Ltd. TTa reports personal fees from Abbvie GK, and grants from Abbvie GK, EA Pharma Co., Ltd, Eisai Co., Ltd., outside the submitted work. TN reports grants from Sekisui Medical Co., Ltd., outside the submitted work. TKa reports research grants from Miyarisan Pharmaceutical Co., Ltd., Ezaki Glico Co., Ltd.; scholarship grants from Abbvie GK, Mochida Pharmaceutical Co., Ltd., Kyorin Pharmaceutical Co., Ltd., Taiho Pharmaceutical Co., Ltd., Daiichisankyo Co., Ltd., Mitsubishi Tanabe Pharma, Takeda Pharmaceutical Company Ltd, JIMRO Co., Ltd., outside the submitted work. TM reports personal fees from Abbvie GK, EA Pharma Co., Ltd, Mitsubishi Tanabe Pharma, Takeda Pharmaceutical Co. Ltd.; grants from Mitsubishi Tanabe Pharma, Nippon Kayaku Co., Ltd., outside the submitted work. The other authors have no conflict of interest to disclose. Kochi University is a Patent holder for LRG.

Ethical approval This study was conducted in accordance with the latest version of the declaration of Helsinki. This study was approved by the Institutional Review Board or the Ethical Committee at each institution.

Informed consent Written informed consent was obtained from all patients.

Open Access This article is licensed under a Creative Commons Attribution 4.0 International License, which permits use, sharing, adaptation, distribution and reproduction in any medium or format, as long as you give appropriate credit to the original author(s) and the source, provide a link to the Creative Commons licence, and indicate if changes were made. The images or other third party material in this article are included in the article's Creative Commons licence, unless indicated otherwise in a credit line to the material. If material is not included in the article's Creative Commons licence and your intended use is not permitted by statutory regulation or exceeds the permitted use, you will need to obtain permission directly from the copyright holder. To view a copy of this licence, visit http://creativecommons. org/licenses/by/4.0/.

\section{References}

1. Ng SC, Shi HY, Hamidi N, et al. Worldwide incidence and prevalence of inflammatory bowel disease in the 21 st century: a systematic review of population-based studies. Lancet. 2018;390:2769-78.

2. Murakami Y, Nishiwaki Y, Oba MS, et al. Estimated prevalence of ulcerative colitis and Crohn's disease in Japan in 2014: an analysis of a nationwide survey. $\mathbf{J}$ Gastroenterol. 2019;54:1070-7.

3. Verstockt B, Ferrante M, Vermeire S, et al. New treatment options for inflammatory bowel diseases. J Gastroenterol. 2018;53:585-90.

4. Turner D, Ricciuto A, Lewis A, et al. STRIDE-II: an update on the selecting therapeutic targets in inflammatory bowel disease (STRIDE) initiative of the international organization for the study of IBD (IOIBD): determining therapeutic goals for treat-to-target strategies in IBD. Gastroenterology. 2021;160:1570-83.

5. Magro F, Gionchetti P, Eliakim R, et al. Third European evidence-based consensus on diagnosis and management of ulcerative colitis. Part 1: definitions, diagnosis, extra-intestinal manifestations, pregnancy, cancer surveillance, surgery, and ileoanal pouch disorders. J Crohns Colitis. 2017;11:649-70.

6. Vermeire S, Van Assche G, Rutgeerts P. Laboratory markers in IBD: useful, magic, or unnecessary toys? Gut. 2006;55:426-31.

7. Falvey JD, Hoskin T, Meijer B, et al. Disease activity assessment in IBD: clinical indices and biomarkers fail to predict endoscopic remission. Inflamm Bowel Dis. 2015;21:824-31.

8. Walsh A, Kormilitzin A, Hinds C, et al. Defining faecal calprotectin thresholds as a surrogate for endoscopic and histological disease activity in ulcerative colitis-a prospective analysis. J Crohns Colitis. 2019;13:424-30.

9. Serada S, Fujimoto M, Ogata A, et al. iTRAQ-based proteomic identification of leucine-rich alpha-2 glycoprotein as a novel inflammatory biomarker in autoimmune diseases. Ann Rheum Dis. 2010;69:770-4.

10. Haupt $H$. Baudner $S$ [Isolation and characterization of an unknown, leucine-rich 3.1-S-alpha2-glycoprotein from human serum (author's transl)]. Hoppe Seylers Z Physiol Chem. 1977;358:639-46.

11. Serada S, Fujimoto M, Terabe F, et al. Serum leucine-rich alpha2 glycoprotein is a disease activity biomarker in ulcerative colitis. Inflamm Bowel Dis. 2012;18:2169-79.

12. Shinzaki S, Matsuoka K, Iijima H, et al. Leucine-rich Alpha-2 glycoprotein is a serum biomarker of mucosal healing in ulcerative colitis. J Crohns Colitis. 2017;11:84-91.

13. Matsuoka K, Kobayashi T, Ueno F, et al. Evidence-based clinical practice guidelines for inflammatory bowel disease. J Gastroenterol. 2018;53:305-53.

14. Daperno M, D'Haens G, Van Assche G, et al. Development and validation of a new, simplified endoscopic activity score for Crohn's disease: the SES-CD. GastrointestEndosc. 2004;60:505-12.

15. Schroeder KW, Tremaine WJ, Ilstrup DM. Coated oral 5 -aminosalicylic acid therapy for mildly to moderately active ulcerative colitis. A randomized study. $N$ Engl J Med. 1987;317:1625-9.

16. Vuitton L, Marteau P, Sandborn WJ, et al. IOIBD technical review on endoscopic indices for Crohn's disease clinical trials. Gut. 2016;65:1447-55.

17. D'Haens G, Sandborn WJ, Feagan BG, et al. A review of activity indices and efficacy end points for clinical trials of medical therapy in adults with ulcerative colitis. Gastroenterology. 2007;132:763-86.

18. Satsangi J, Silverberg MS, Vermeire S, et al. The Montreal classification of inflammatory bowel disease: controversies, consensus, and implications. Gut. 2006;55:749-53.

19. Smids C, Horjus T, Horje CS, Nierkens S, et al. Candidate serum markers in early crohn'sdisease:predictors of disease course. J Crohns Colitis. 2017;11:1090-100.

20. Shinzaki S, Kuroki E, Iijima H, et al. Lectin-based immunoassay for aberrant IgG glycosylation as the biomarker for Crohn's disease. Inflamm Bowel Dis. 2013;19:321-31.

21. Colombel JF, Panaccione R, Bossuyt P, et al. Effect of tight control management on Crohn's disease (CALM): a multicentre, randomised, controlled phase 3 trial. Lancet. 2018;390:2779-89.

22. Sands BE. Biomarkers of inflammation in inflammatory bowel disease. Gastroenterology. 2015;149(1275-1285):e2.

23. Schoepfer AM, Beglinger C, Straumann A, et al. Fecalcalprotectin more accurately reflects endoscopic activity of ulcerative colitis than the Lichtiger Index, C-reactive protein, platelets, 
hemoglobin, and blood leukocytes. Inflamm Bowel Dis. 2013;19:332-41.

24. Iwamoto F, Matsuoka K, Motobayashi M, et al. Prediction of disease activity of Crohn's disease through fecalcalprotectin evaluated by balloon-assisted endoscopy. J Gastroenterol Hepatol. 2018;33:1984-9.

25. Kawashima K, Ishihara S, Yuki T, et al. Fecalcalprotectin more accurately predicts endoscopic remission of crohn's disease than serological biomarkers evaluated using balloon-assisted enteroscopy. Inflamm Bowel Dis. 2017;23:2027-34.
26. Arai T, Takeuchi K, Miyamura M, et al. Level of fecalcalprotectin correlates with severity of small bowel crohn's disease, measured by balloon-assisted enteroscopy and computed tomography enterography. ClinGastroenterolHepatol. 2017;15:56-62.

Publisher's Note Springer Nature remains neutral with regard to jurisdictional claims in published maps and institutional affiliations. 